Proc. 15th Int. Conference on Defects Recognition, Imaging and Physics in Semiconductors, Warsaw, Poland 2013

\title{
A New Generation of Variable Temperature Scanning Probe Microscope for Spectroscopy
}

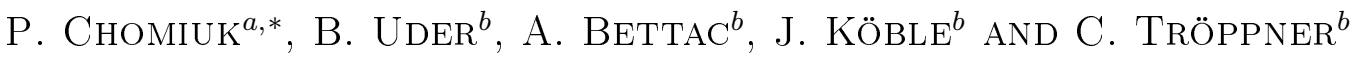 \\ ${ }^{a}$ APVACUUM Ltd., Klonowa 24, 62-002 Suchy Las k. Poznania, Poland \\ ${ }^{b}$ Oxford Instruments Omicron NanoScience, Limburger Str. 75, 65232 Taunusstein, Germany
}

\begin{abstract}
In this contribution we present the design and first results of a new generation of variable temperature scanning probe microscope that has been developed to enhance the performance in tunnelling spectroscopy at lower temperatures. Its performance has been proven with imaging and spectroscopy experiments on the well known $\mathrm{Si}(111), \mathrm{Au}(111)$, and $\mathrm{Ag}(111)$ surfaces.
\end{abstract}

DOI: 10.12693/APhysPolA.125.1049

PACS: 68.37.Ef, 73.20.At

\section{Introduction}

Conventional variable temperature scanning probe microscopes (SPMs) became available about 20 years ago. The main idea was to look at structures at lower temperatures $(\approx 50 \mathrm{~K})$ where the thermal mobility is reduced and imaging of structures (i.e. molecules on a surface) was easier to achieve. Conventional variable temperature SPM microscopes [1-3] always cool the sample only. The rest of the instrument, including the scanner with the tunnelling tip, stays at room temperature. This design has a lot of advantages with respect to flexibility and an open design. However, the sample is always faced a warm tip and the instrument needs a careful design to minimize thermal drift. With this design approach it has been possible to reduce the remaining thermal drift to values lower than $50 \mathrm{pm} / \mathrm{s}$ [1]. Imaging surfaces at low temperatures deliver good results, however this setup is not ideal for spectroscopy measurements due to the remaining drift between the tip and the surface. In conventional variable temperature designs it is therefore required to perform spectroscopy experiments quickly.

In the last decade tunnelling spectroscopy has received more and more importance in surface science. In addition to imaging a surface or a surface with molecular structures, tunnelling spectroscopy is required to learn more about these new structures and their interaction with the surface or the environment. Due to the lack of spectroscopy performance of conventional variable temperature designs, research moved to low temperature scanning tunneling microscopy (STM) designs where the complete STM is kept at a low temperature ( $4 \mathrm{~K}$ or $77 \mathrm{~K}$ ) by using bath cryostats. However, these instruments often require a system solution due to their complex design with shielded nitrogen and helium tanks $[4,5]$.

*corresponding author; e-mail: p.chomiuk@apvacuum.com
An alternative and a next generation is a compact variable temperature SPM with a cold tip. This needs a new approach, the design of a new and compact SPM stage, optimized thermal shields, a dedicated heat flow control and a stable and precise temperature regulation.

\section{Experimental}

The new SPM is based on a novel design which uses a new flow cryostat compatible for cooling with liquid nitrogen or helium. In contrast to earlier established designs of variable temperature SPM, where only the sample is cooled, this new SPM also cools the scanner and tip. This is realised by a newly developed compact and stable SPM stage with thermal shields and a dedicated cooling management system. With this design we achieve lower temperatures and improve drift by more than an order of magnitude compared to previous variable temperature stages. Sample temperatures down to $10 \mathrm{~K}$ (with helium) and $95 \mathrm{~K}$ (with nitrogen) have been achieved. The temperature stability is better than $5 \mathrm{mK} / \mathrm{min}$ and the measured thermal drift is below $1 \mathrm{pm} / \mathrm{s}$. During cooling the mechanical $z$ stability is better than $3 \mathrm{pm}$. These conditions offer enhanced spectroscopy measurement capability with a cold tip. "Loop off" times of up to $10 \mathrm{~s}$ per single spectroscopy curve have been measured. The new flow cryostat also allows for changing between nitrogen cooling and helium cooling in less than 90 min during a running experiment. Pre-cooling with nitrogen during the starting phase of an experiment also reduces running costs for liquid helium. This new SPM is configured for imaging in STM as well as AFM with a non-optical sensor in a temperature range between 15 and $400 \mathrm{~K}$. Switching between the 2 modes can be accomplished without breaking vacuum.

\section{Results}

The Fermi SPM was tested at full cooling power using the flow cryostat with liquid helium. First experiments were done on the well known $\operatorname{Si}(111) 7 \times 7$ reconstruction 
(Fig. 1) at $10 \mathrm{~K}$. The image shows clear atomic resolution of the topography with characteristic rosettes and missing atoms (left), and a line profile (right). This image corresponds well to examples presented in literature $[6-8]$.
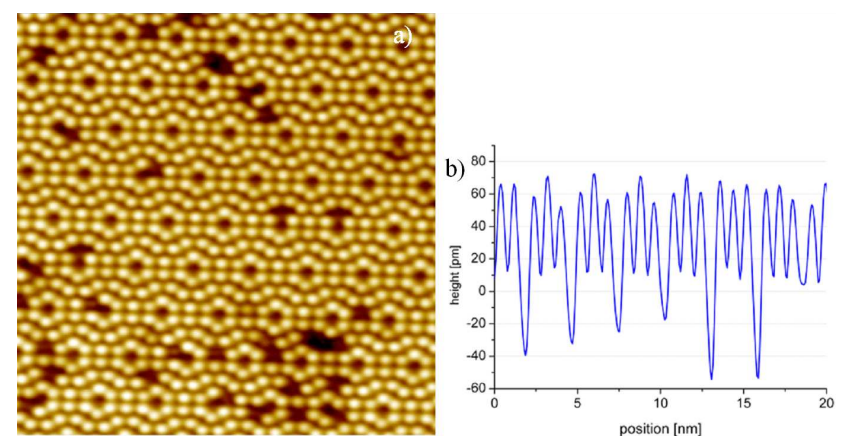

Fig. 1. $\operatorname{Si}(111) 7 \times 7$ reconstruction, $20 \mathrm{~nm} \times 20 \mathrm{~nm}$, $U_{\text {gap }}=1.4 \mathrm{~V}, I_{\mathrm{t}}=1.5 \mathrm{nA}$, sample stage at $10 \mathrm{~K}$, tip scanner at $25 \mathrm{~K}(\mathrm{a})$, line profile (b).
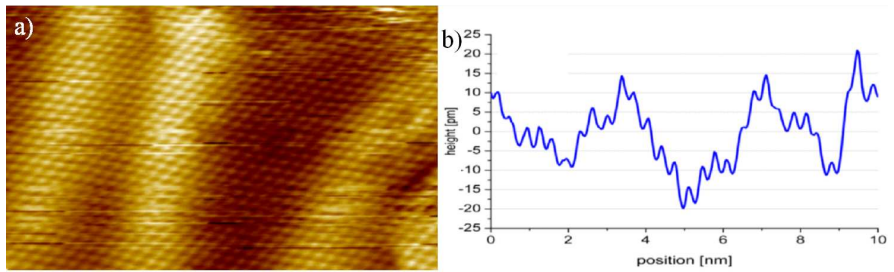

Fig. 2. $\mathrm{Au}(111)$ topography $10 \mathrm{~nm} \times 6 \mathrm{~nm}, U_{\text {gap }}=$ $-0.5 \mathrm{~V}, I_{\mathrm{t}}=2 \mathrm{nA}$, sample stage at $10 \mathrm{~K}$, tip/scanner at $25 \mathrm{~K}$, unfiltered raw data (a), line profile proving $z$ stability (b).

Similarly, an Au(111) surface was imaged at $10 \mathrm{~K}$. Figure 2 shows topography with atomic resolution. Unfiltered raw data is presented. The line profile is a proof for the high resolution capability under these conditions. $\mathrm{Au}$ corrugation is in the range of $5 \mathrm{pm}$ and corresponds well with the literature data [9]. We estimate the $z$ stability of only a few pm with full cooling power. Low thermal drift has been also confirmed by spectroscopy experiments.

The performance for tunnelling spectroscopy was tested on $\mathrm{Au}(111)$ surface at a sample temperature of $10 \mathrm{~K}$ (Fig. 3). Having tip and sample cold results in a low thermal $z$ drift and also piezo creeping effects are reduced with the cold scanner. After positioning of the tip on the surface in regulated mode the feedback loop was switched off. The gap voltage was ramped from $-0.2 \mathrm{~V}$ to $0.8 \mathrm{~V}$ and back to $-0.2 \mathrm{~V}$. The total time for a single measurement was $10 \mathrm{~s}$. Both $I(V)$ and $\mathrm{d} I / \mathrm{d} V$ characteristics were measured simultaneously. The $I(V)$ characteristic for ramp up and ramp down only shows a small offset. The drift in $z$ direction is estimated in below $1 \mathrm{pm} / \mathrm{s}$. Also seen in the $\mathrm{d} I / \mathrm{d} V$ spectrum (blue) is the
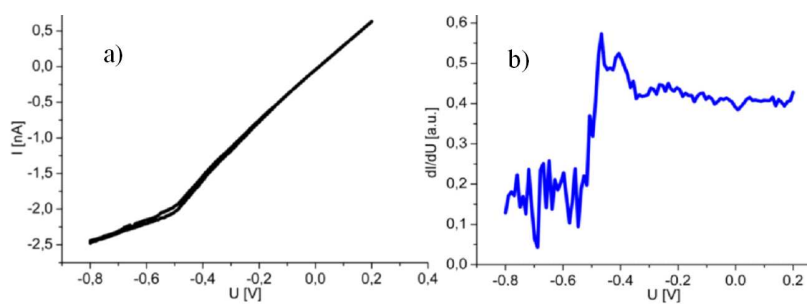

Fig. 3. Point spectroscopy characteristic on the $\mathrm{Au}(111)$. Voltage ramped from $-0.2 \mathrm{~V}$ to $0.8 \mathrm{~V}$ to -0.2 V. Feedback loop switched off for $10 \mathrm{~s}$ (a), simultaneously recorded $\mathrm{d} I / \mathrm{d} V$ characteristic showing the surface state on $\mathrm{Au}(111)$ (b).

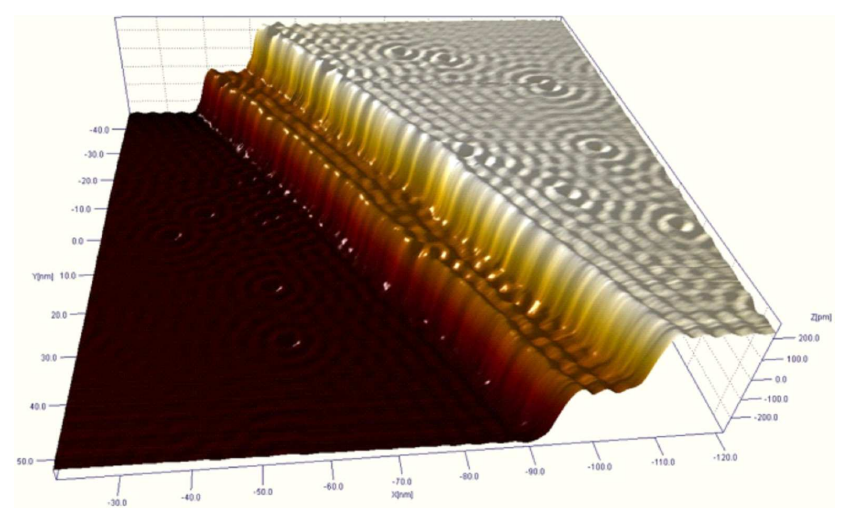

Fig. 4. Electron standing waves on the $\mathrm{Ag}(111)$, scan range: $100 \mathrm{~nm} \times 100 \mathrm{~nm}, U_{\text {gap }}=5 \mathrm{mV}, I_{\mathrm{t}}=2 \mathrm{nA}$, sample stage at $10 \mathrm{~K}$, tip/scanner at $25 \mathrm{~K}$.

onset of the surface states on the $\mathrm{Au}(111)$ surface which agrees with the value in literature [10].

Standing electron waves have been observed on $\operatorname{Ag}(111)$ surface at $10 \mathrm{~K}$ (Fig. 4). The $\mathrm{Ag}(111)$ surface has a surface state around $-65 \mathrm{mV}$ which forms a two-dimensional nearly free electron gas parallel to the surface and many physical phenomena may be observed [11-13]. The surface state of $\mathrm{Ag}(111)$ can be directly measured using $\mathrm{d} I / \mathrm{d} V$ spectroscopy. These sur-

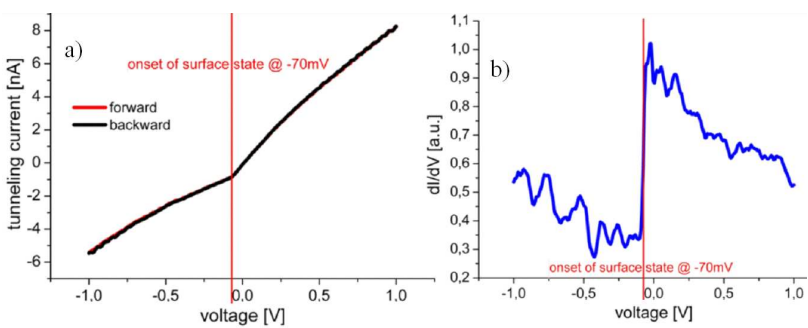

Fig. 5. Point spectroscopy characteristic, voltage ramped from $-1 \mathrm{~V}$ to $1 \mathrm{~V}$ to $-1 \mathrm{~V}$. $I(V)$ characteristics show onset of the surface state at approximately $-70 \mathrm{mV}$ (a), simultaneously measured $\mathrm{d} I / \mathrm{d} V$ signal (b). 
face state electrons scatter from defects and step edges on $\mathrm{Ag}(111)$ and produce interference patterns known as electron standing waves [14]. The electron standing waves can be directly observed even in normal STM images when acquired at lower biases close to the Fermi energy on $\mathrm{Ag}(111)$. The characteristics of $I / V$ and $\mathrm{d} I / \mathrm{d} V$ spectroscopy are shown in Fig. 5.

\section{Summary}

We successfully realised a new generation of variable temperature SPM with a cold tip. The "Fermi SPM" uses a flow cryostat that can be operated with liquid helium or liquid nitrogen. Sample and scanner are individually connected to the cryostat cold finger using flexible cooling connections. Sample and scanner temperature are individually regulated. Sample temperature of $10 \mathrm{~K}$ has been achieved with helium cooling, and $95 \mathrm{~K}$ with nitrogen cooling. $Z$ stability is better than $3 \mathrm{pm}$ during cooling. Thermal drift is below $1 \mathrm{pm} / \mathrm{s}$. Temperature stability is better than $5 \mathrm{mK} / \mathrm{min}$. The device may be operated in the temperature range $10-400 \mathrm{~K}$. The microscope performance has been proven with well known $\mathrm{Si}(111), \mathrm{Au}(111)$, and $\mathrm{Ag}(111)$ surfaces.

\section{References}

[1] Omicron VT SPM.

[2] RHK Variable Temp BEETLE.

[3] SPM 150 Aarhus, SPM 250 Aarhus EVT.

[4] Omicron LT SPM.

[5] Crea-Tec LT STM.

[6] G. Binnig, H. Rohrer, Ch. Gerber, E. Weibel, Phys. Rev. Lett. 50, 120 (1983).

[7] F.J. Giessibl, S. Hembacher, H. Bielefeldt, J. Mannhart, Science 289, 422 (2000).

[8] A.J. Weymouth, T. Wutscher, J. Welker, T. Hofmann, F.J. Giessibl, Phys. Rev. Lett. 106, 226801 (2011).

[9] Ch. Woell, S. Chiang, R.J. Wilson, P.H. Lippel, Phys. Rev. B 39, 7988 (1989).

[10] W. Chen, V. Madhavan, T. Jamneala, M.F. Crommie, Phys. Rev. B 80, 1469 (1998).

[11] J. Li, W.-D. Schneider, R. Berndt, Phys. Rev. B 56 , 7656 (1997).

[12] L. Bürgi, O. Jeandupeux, A. Hirstein, H. Brune, K. Kern, Phys. Rev. Lett. 81, 5370 (1998).

[13] K. Morgenstern, K.-F. Braun, K.-H. Rieder, Phys. Rev. Lett. 89, 226801 (2002).

[14] R.M. Feenstra, Saw Wai Hla, Chapter from LandoltBörnstein Review: Scanning Tunneling Microscopy, to be published in Landolt-Börnstein New Series, 2014 . 